\title{
Exhaust Condensate Treatment Technology Based on Acoustic Levitation
}

\author{
Fuqiang Zhang, Zhao Guan, Hao Jiang \\ School of Mechanical and Automotive Engineering, Shanghai University of Engineering Science, Shanghai, China \\ Email: 1360199980@qq.com
}

How to cite this paper: Zhang, F.Q., Guan, Z. and Jiang, H. (2018) Exhaust Condensate Treatment Technology Based on Acoustic Levitation. Open Access Library Journal, 5: e5097.

https://doi.org/10.4236/oalib.1105097

Received: December 4, 2018

Accepted: December 17, 2018

Published: December 20, 2018

Copyright (c) 2018 by authors and Open Access Library Inc.

This work is licensed under the Creative

Commons Attribution International

License (CC BY 4.0).

http://creativecommons.org/licenses/by/4.0/

\section{cc) (i) Open Access}

\begin{abstract}
In view of the condensed water droplets falling into the ground in winter, the automobile exhaust pipe in the cold area brings hidden dangers to the urban traffic safety. The research on the development of the car exhaust pipe condensate on-board treatment device is carried out. The reaction process of engine combustion and the main components of exhaust gas were analyzed. The working principle of ultrasonic suspension device was introduced. The temperature of automobile exhaust gas was measured by experiment. The COMSOL particle tracking module was used to simulate the acoustic suspension exhaust gas.
\end{abstract}

\section{Subject Areas}

Mechanical Engineering

\section{Keywords}

Exhaust Pipe, Condensed Water, Acoustic Suspension, Simulation

\section{Introduction}

During the driving process of the winter car, there is liquid dripping at the exit of the exhaust pipe, and the water droplets are easy to form points, sheets, and linear ice surfaces, which are particularly obvious in the traffic jams, crossroads, and uphill sections of urban traffic peak vehicles. The ice surface will increase the driving braking distance and bring traffic safety hazards, which is one of the reasons why the probability of winter traffic accidents is higher than other seasons. In addition, the mixing of water droplets and exhaust gas formed at the tail of the exhaust pipe of the automobile can cause serious corrosion to the exhaust pipe of the automobile and shorten the service life of the exhaust pipe of the au- 
tomobile [1]. To solve this problem, the designer adds an installation angle when installing the exhaust pipe. Reducing the degree of corrosion of the exhaust pipe, the traditional method does not seem to fundamentally solve the problem caused by exhaust condensate. This paper envisages that the use of ultrasonic suspension to control the exhaust gas for suspension discharge, inhibit the formation of condensed water and discharge as much as possible in gaseous form will fundamentally solve the harm caused by it.

\section{Main Components of the Combustion Process and Exhaust}

Gasoline is mainly composed of two elements, $\mathrm{C}$ and $\mathrm{H}$, followed by a small amount of oxygen, fluorine and sulfur. The mass ratio of $\mathrm{C}$ and $\mathrm{H}$ in domestic gasoline is $0.875: 0.125$. Assuming that the average molecular composition is a general-purpose carbon hydroxide fuel, according to the conservation of atomic number and the law of conservation of mass, the chemical equation of the fuel is completely burned [1] as follow [2]:

$\mathrm{C}_{x} \mathrm{H}_{y} \mathrm{O}_{z}+\left(x+\frac{y}{4}-\frac{z}{2}\right)\left(\mathrm{O}_{2}+3.773 \mathrm{~N}_{2}\right)=x \mathrm{CO}_{2}+\frac{y}{2} \mathrm{H}_{2} \mathrm{O}+3.772\left(x+\frac{y}{4}-\frac{z}{2}\right) \mathrm{N}_{2}$

The density of gasoline is $0.725 \mathrm{~g} / \mathrm{ml}$, and the theoretical calculation of the product of $1 \mathrm{~kg}$ of gasoline when fully burned is calculated by the principle of mass conservation.

$$
\begin{gathered}
n_{(c)}=\frac{m(\mathrm{C})}{M(\mathrm{C})}=\frac{0.875}{12}=0.0729(\mathrm{~mol})=n\left(\mathrm{CO}_{2}\right) \\
m\left(\mathrm{CO}_{2}\right)=n\left(\mathrm{CO}_{2}\right) \times M\left(\mathrm{CO}_{2}\right)=0.0729 \times 44=3.208(\mathrm{~kg})
\end{gathered}
$$

Then, the theoretical amount of $\mathrm{CO}_{2}$ produced by $1 \mathrm{~L}$ gasoline when fully burned is $2.273 \mathrm{~kg}$. Similarly, the theoretical calculation of the product of $\mathrm{H}_{2} \mathrm{O}$ when $1 \mathrm{~L}$ gasoline is fully burned is $0.906 \mathrm{~kg}$.

\section{Principle of Ultrasonic Suspension Device}

The ultrasonic suspension technology uses the high-frequency piston vibration of the radiation end of the ultrasonic transducer to generate ultrasonic waves. The ultrasonic waves propagate through the medium, and the reflection end is placed on the propagation path, and the distance between the radiation end surface and the reflection end surface is adjusted to be an integer of half wavelength of the ultrasonic wave. When doubled, the incident wave and the reflected wave are repeatedly superimposed in the sound field space to form a high-strength standing wave sound field, and the object placed in the standing wave sound field is suspended by the action of the radiation sound pressure. Therefore, the experimental device needs to be installed at the radiant end of the exhaust pipe of the automobile. The vibration acceleration of transducer is $a$, and the reflecting end is installed at the other end, and the ultrasonic frequency selected is $f$, the speed of sound waves in the medium is $c$, according to the formula [3]. 


$$
\lambda=\frac{c}{f}
$$

And when the distance between the radiation end and the reflection end is satisfied [4]:

$$
H=n * \lambda / 2, \quad n=1,2,3 .
$$

There will be resonance, and the standing wave node can suspend the object as follow Figure 1.

Sound radiation force [5]:

$$
F=\frac{5}{6} \pi \rho_{0} A^{2} r^{3} \sin (2 k h)
$$

where $\rho_{0}$ is the density of the medium, $A$ is the amplitude, and $r$ is the radius of the suspended object, $\mathrm{h}$ is the distance from the center of the ball to the vertical direction of the transmitting end; Thus the density is an important factor affecting the acoustic suspension capability.

\section{Speed of Sound Changes with Temperature}

When the temperature is added to the ultrasonic levitation, it not only affects the density of the medium, but also affects the speed of sound propagation, thereby affecting the magnitude of the acoustic levitation force. The function expression of the radiation force $F$ after the temperature change [6].

$$
F=395 p^{2} r^{3} A^{2} f \frac{r_{0}}{(273+t)\left(c_{0}+0.6 t\right)}
$$

The unknown is only the temperature $t$. After the function is obtained, the image is drawn as shown in Figure 2. It can be seen from the figure that after considering the influence of temperature on the density of the medium and the speed of sound propagation, the relationship between the acoustic radiation force and the temperature is increased with the increase of temperature, although the acoustic radiation is increased with the increase of temperature. The force is reduced [6]. Since the exhaust gas is in the form of gas, a small radiation force can have a working effect, so temperature is not the most important factor.

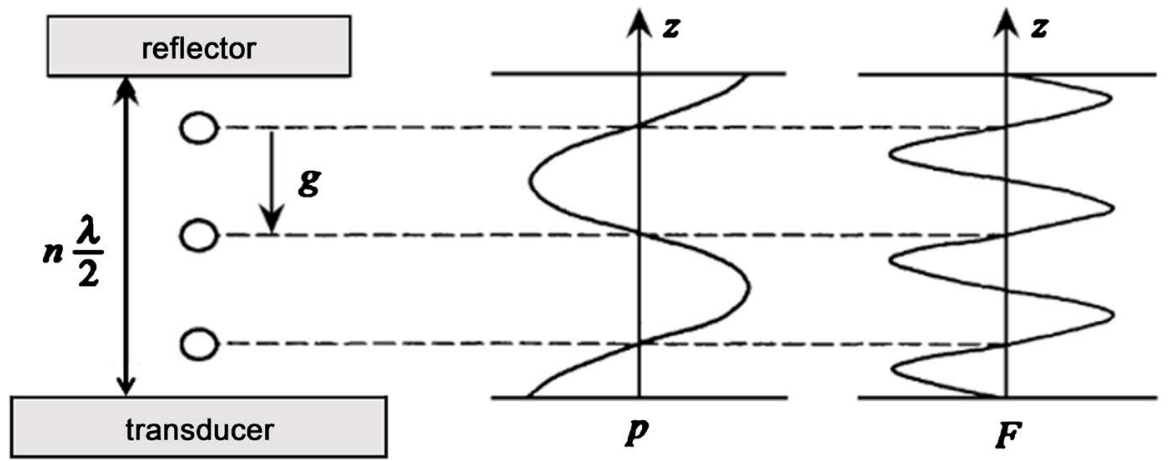

Figure 1. Acoustic suspension schematic. 


\section{Modeling Simulation}

A model for the emission control of acoustic suspension control is established by COMSOL. The device should be installed at the end of the exhaust system, with an ultrasonic transducer installed below, and a reflective section at the top of the other side. The simplified model is as follows Figure 3.

The sound pressure field module and the particle tracking module are set, and the medium density is set to 2 , and the temperature affects the floating force by changing the medium to the sound wave speed, so the sound wave speed is 400 , and the frequency of the transducer is $28 \mathrm{kHz}$, the vibration of acceleration $a_{0}=1.5 \times 10^{6} \mathrm{~m} / \mathrm{s}^{2}$, the boundary condition of the reflective end face is set as a hard reflection surface, and the boundary conditions on both sides are set as soft boundary conditions.

The meshing of the model is as follows Figure 4 .

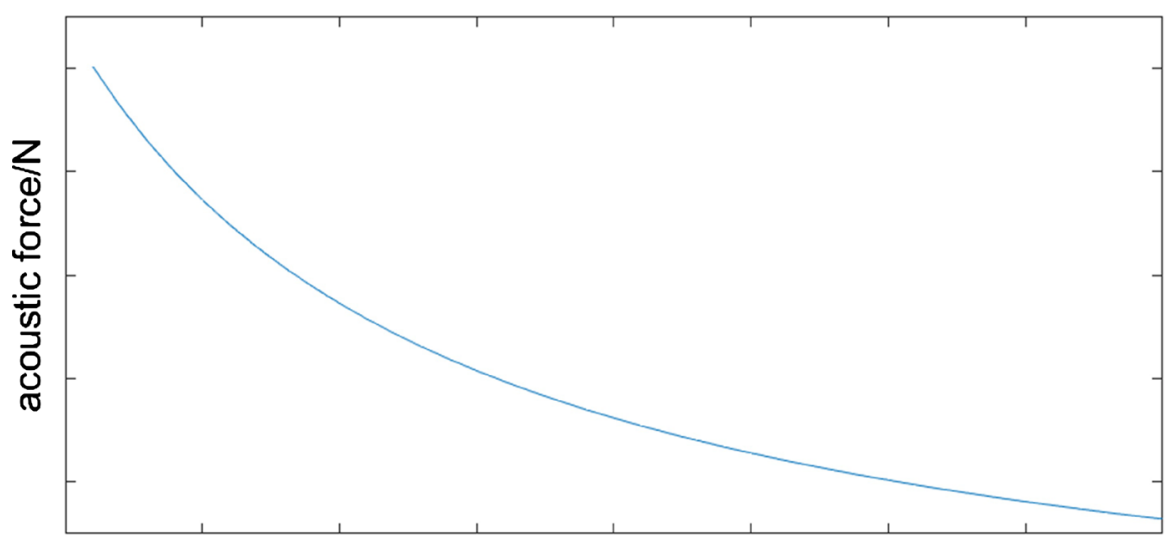

Temperature

Figure 2. Acoustic force with temperature.

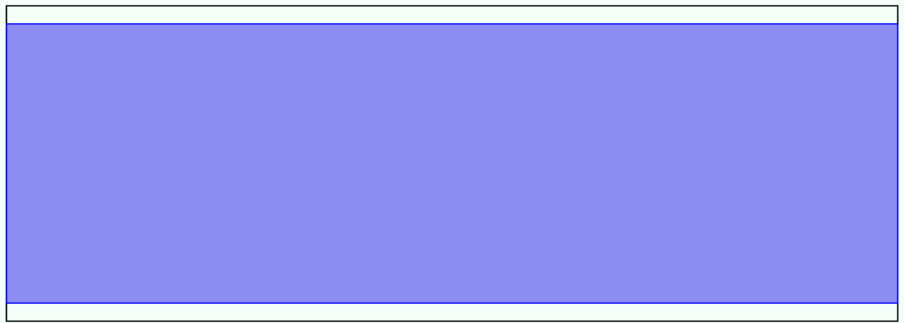

Figure 3. Mathematical model.

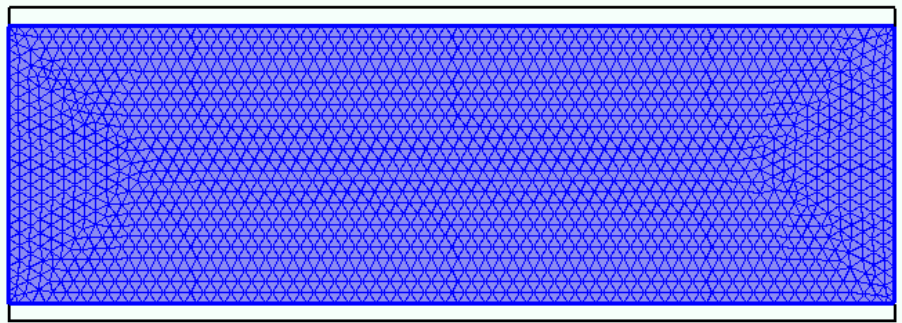

Figure 4. Meshing. 
According to the results of the transient calculation, according to the simulation results Figure 5, it can be seen that the particles are stably layered due to the sound pressure radiation, and the number of layers is simulated by the cavity $\mathrm{H}$ and the natural frequency of the transducer vibrator is determined.

Partial magnification as shown in Figure 6.

\section{Conclusion}

It is found through simulation that the ultrasonic suspension suspends the small particles of the exhaust gas and produces stratification, which can be applied to the exhaust emissions of automobiles. Even if the temperature generated by the automobile exhaust is high, after passing through multiple parts of the exhaust system, the temperature significantly reduced, can adapt to the effect of acoustic suspension. Temperature-to-ultrasonic levitation changes the acoustic radiation force by affecting the speed of sound wave propagation. Since the application we use is an open space rather than a closed space, it is known that the temperature change in the closed space affects the density of the gas, and the density. The change is great for changing the acoustic suspension characteristics. The change in open space temperature has no effect on density, only the wave speed is changed.

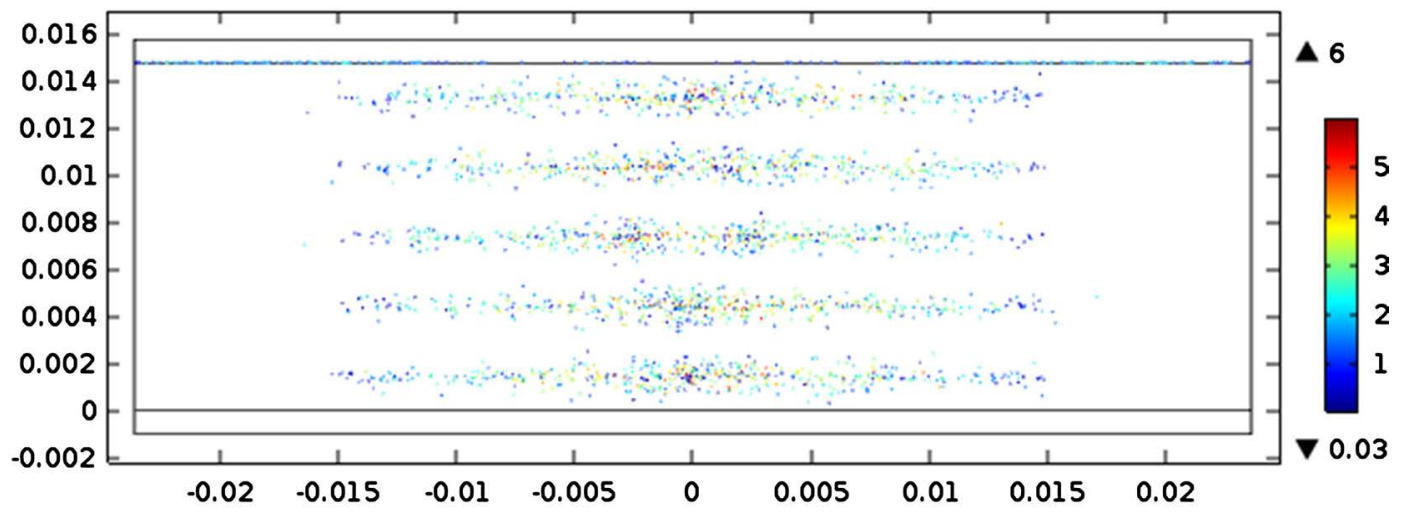

Figure 5. Particle motion trajectory.

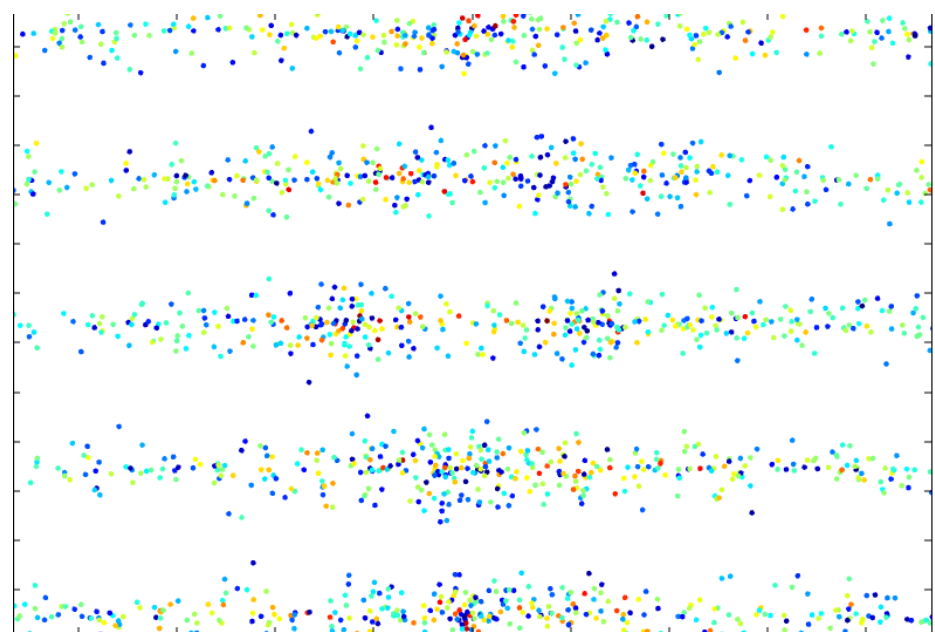

Figure 6. Partial magnification. 


\section{Conflicts of Interest}

The authors declare no conflicts of interest regarding the publication of this paper.

\section{References}

[1] Zhang, L., Zang, J. and Liu, Q.G. (2008) Feasibility Analysis of Controlling the Moisture Existed in Exhaust Gas of Ethanol Gasoline Car in Cool Area. Communications Standardization.

[2] Bao, Y., Tian, F., An, Y.D., et al. (2015) Experimental Study of Automobile Emission Pipes Freeze Femperature Spread in Winter. Technology \& Economy in Areas of Communications.

[3] Brandt, E.H. (1989) Levitation in Physics. Science, 243, 349-355. https://doi.org/10.1126/science.243.4889.349

[4] Xie, W.J. (2002) Latest Progress in Acoustic Levitation. Physics.

[5] Yosioka, K. and Kawasima, Y. (1955) Acoustic Radiation Pressure on a Compressible Sphere. Acta Acustica United with Acustica, 167-173.

[6] Trinh, E.H. and Ohsaka, K. (1995) Measurement of Density, Sound Velocity, Surface Tension, and Viscosity of Freely Suspended Supercooled Liquids. International Journal of Thermophysics. 